\title{
Calculation of the longitudinal emittance dilution in a RF cavity
}

FERMILAB-TM- -2736-AD

May 6, 2020

\author{
A. Shemyakin, Fermilab, Batavia, IL 60510, USA \\ shemyakin@fnal.gov
}

Abstract

Dilution of the longitudinal rms emittance of a bunch passing a RF cavity is estimated for the sinusoidal dependence of the energy gain on phase and Gaussian beam distribution.

\section{Model}

Dilution of the longitudinal rms emittance of a bunch passing a RF cavity can be estimated caused in a simplest model:

a) Energy $\Delta E$ gained by a particle depends only on its phase $\varphi$ and the energy gain on crest $U$ as

$$
\Delta E=U \cdot \cos \varphi .
$$

b) The energy gain of all particles is small in comparison with the total kinetic energy

$$
U \ll(\gamma-1) M c^{2} \text {, }
$$

where $\gamma$ is the relativistic factor, $M$ is particle mass, and $c$ is the speed of light.

c) The longitudinal distribution is Gaussian

$$
\rho\left(z, z^{\prime}\right) \equiv \frac{d^{2} N}{d z d z^{\prime}}=\frac{N_{0}}{2 \pi \varepsilon} e^{-\frac{J}{\varepsilon_{0}}}, J=\frac{1}{2}\left(\gamma_{T} z^{2}+2 \alpha_{T} z \cdot z^{\prime}+\beta_{T} z^{\prime 2}\right),
$$

where $N_{0}$ is the total number of particles in the bunch, $\varepsilon_{0}$ is the rms longitudinal emittance and $\alpha_{T}, \beta_{T}, \gamma_{T}$ are the longitudinal Twiss functions. The distribution is expressed in terms of the distance to the synchronous particle $z$ and the rate of changing this distance along the longitudinal coordinate $S$,

$$
z^{\prime} \equiv \frac{d z}{d s}=-\frac{\Delta \beta}{\beta},
$$

where $\beta c$ is the velocity of the synchronous particle and $\Delta \beta c$ is the velocity deviation.

d) Effects of space charge, beam loading, etc. are ignored. The only considered effect is the different energy gain for particles with different $z$.

\section{Calculation}

After passing the cavity, the particle velocity with respect to the synchronous particle changes by $\delta \beta c$, so that 


$$
\begin{gathered}
z_{1}^{\prime}=z^{\prime}-\frac{\delta \beta}{\beta}=z^{\prime}-\frac{1}{\beta^{2} \gamma^{3}} \frac{\Delta E-U \cdot \cos \varphi_{s}}{M c^{2}} \equiv z^{\prime}+\delta z^{\prime}, \\
\delta z^{\prime}(z)=-\frac{1}{\beta^{2} \gamma^{3}} \frac{U}{M c^{2}}\left[\cos \left(\varphi_{s}-\Delta \varphi\right)-\cos \varphi_{s}\right], \\
\Delta \varphi=\frac{2 \pi f_{c} z}{\beta c}
\end{gathered}
$$

where $f_{c}$ is the cavity frequency, and $\varphi_{s}$ is the synchronous phase.

The diluted rms emittance $\varepsilon_{1}$ is calculated by usual averaging over all particles:

$$
\begin{gathered}
\varepsilon_{1}^{2}=\overline{z^{2}} \overline{{z_{1}^{\prime}}^{2}}-{\overline{z z_{1}^{\prime}}}^{2}=\overline{z^{2}}\left(\overline{{z^{\prime}}^{2}}+2 \overline{z^{\prime} \delta z^{\prime}}+\overline{\delta{z^{\prime}}^{2}}\right)-\left({\overline{z z^{\prime}}}^{2}+2 \overline{z z^{\prime}} \cdot \overline{z \delta z^{\prime}}+{\overline{z \delta z^{\prime}}}^{2}\right)= \\
=\varepsilon_{0}^{2}+\sigma_{z}^{2} \cdot 2 \overline{z^{\prime} \delta z^{\prime}}+\sigma_{z}^{2} \cdot \overline{\delta z^{\prime 2}}-2 \overline{z z^{\prime}} \cdot \overline{z \delta z^{\prime}}-{\overline{z \delta z^{\prime}}}^{2}, \\
\varepsilon_{0}^{2} \equiv \overline{z^{2}} \overline{{z^{\prime}}^{2}}-\overline{z z^{\prime}}, \quad \sigma_{z}^{2} \equiv \overline{z^{2}}=\beta_{T} \varepsilon_{0}, \quad \overline{z z^{\prime}}=-\alpha_{T} \varepsilon_{0} .
\end{gathered}
$$

Averaging of each component of Eq.(6) can be made by explicit integration using Eq.(1), Eq.(3), and Eq.(5). The first component is integrated as follows.

$$
\overline{z^{\prime} \delta z^{\prime}}=\frac{1}{N_{0}} \int_{-\infty}^{\infty} d z \int_{-\infty}^{\infty} z^{\prime} \delta z^{\prime} \rho\left(z, z^{\prime}\right) d z^{\prime} .
$$

To simplify the expression, the $z, z^{\prime}$ variables are expressed through the variables $u, v$ :

$$
z=u \sigma_{z} \equiv u \sqrt{\beta_{T} \varepsilon_{0}}, z^{\prime}=\frac{v-\alpha_{T} u}{\sqrt{\frac{\beta_{T}}{\varepsilon_{0}}}},
$$

Eq.(7) can be re-written as

$$
\overline{z^{\prime} \delta z^{\prime}}=\frac{1}{2 \pi} \sqrt{\frac{\varepsilon_{0}}{\beta_{T}}} \int_{-\infty}^{\infty} \delta z^{\prime}(u) d u \int_{-\infty}^{\infty}\left(v-\alpha_{T} u\right) e^{-\frac{u^{2}+v^{2}}{2}} d v=-\frac{\alpha_{T}}{\sqrt{2 \pi}} \sqrt{\frac{\varepsilon_{0}}{\beta_{T}}} \int_{-\infty}^{\infty} \delta z^{\prime}(u) u e^{-\frac{u^{2}}{2}} d u \text {. }
$$

Substituting Eq.(8) to Eq.(5),

$$
\begin{gathered}
\delta z^{\prime}(u)=-\frac{1}{\beta^{2} \gamma^{3}} \frac{U}{M c^{2}}\left[\cos \left(\varphi_{s}-k u\right)-\cos \varphi_{s}\right]=A\left(\cos \varphi_{s} \cos k u+\sin \varphi_{s} \sin k u-\cos \varphi_{s}\right), \\
A \equiv-\frac{1}{\beta^{2} \gamma^{3}} \frac{U}{M c^{2}}, \quad k \equiv \frac{2 \pi f_{c} \sigma_{z}}{\beta c}
\end{gathered}
$$

Integral of the first and third terms in Eq. (10) is zero by parity, and Eq. (9) yields

$$
\overline{z^{\prime} \delta z^{\prime}}=-\frac{\alpha_{T}}{\sqrt{2 \pi}} \sqrt{\frac{\varepsilon_{0}}{\beta_{T}}} A \sin \varphi_{s} \int_{-\infty}^{\infty} \sin k u u e^{-\frac{u^{2}}{2}} d u=-\alpha_{T} \sqrt{\frac{\varepsilon_{0}}{\beta_{T}}} A \sin \varphi_{s} k e^{-\frac{k^{2}}{2}}
$$

Integration of other terms of Eq.(6) is similar:

$$
\begin{gathered}
\overline{\delta z^{\prime 2}}=\frac{1}{2 \pi} \int_{-\infty}^{\infty} \delta z^{\prime 2}(u) d u \int_{-\infty}^{\infty} e^{-\frac{u^{2}+v^{2}}{2}} d v= \\
=\frac{A^{2}}{\sqrt{2 \pi}} \int_{-\infty}^{\infty}\left[1+\frac{\cos 2 \varphi_{s}}{2}+\frac{1}{2} \cos 2 \varphi_{s} \cos 2 k u-\left(1+\cos 2 \varphi_{s}\right) \cos k u\right] e^{-\frac{u^{2}}{2}} d u=
\end{gathered}
$$




$$
\begin{gathered}
=A^{2}\left[1+\frac{\cos 2 \varphi_{s}}{2}+\frac{\cos 2 \varphi_{s} e^{-2 k^{2}}}{2}-\left(1+\cos 2 \varphi_{s}\right) e^{-\frac{k^{2}}{2}}\right], \\
\overline{z \delta z^{\prime}}=\frac{\sigma_{z}}{2 \pi} \int_{-\infty}^{\infty} u \cdot \delta z^{\prime}(u) d u \int_{-\infty}^{\infty} e^{-\frac{u^{2}+v^{2}}{2}} d v=A \sigma_{z} \sin \varphi_{s} k e^{-\frac{k^{2}}{2}} .
\end{gathered}
$$

Substitution of Eq.(11) - (13) into Eq. (6) gives

$$
\begin{gathered}
\varepsilon_{1}^{2}-\varepsilon_{0}^{2}=-2 \sigma_{z}^{2} \alpha_{T} \sqrt{\frac{\varepsilon_{0}}{\beta_{T}}} A \sin \varphi_{s} k e^{-\frac{k^{2}}{2}}+ \\
+A^{2} \sigma_{z}^{2}\left[1+\frac{\cos 2 \varphi_{s}}{2}+\frac{\cos 2 \varphi_{s} e^{-2 k^{2}}}{2}-\left(1+\cos 2 \varphi_{s}\right) e^{-\frac{k^{2}}{2}}\right]+ \\
+2 \alpha_{T} \varepsilon_{0} A \sigma_{z} \sin \varphi_{s} k e^{-\frac{k^{2}}{2}}-\left(A \sigma_{z} \sin \varphi_{s} k e^{-\frac{k^{2}}{2}}\right)^{2} .
\end{gathered}
$$

After combining the terms in Eq. (14), the final expression for the diluted emittance is

$$
\varepsilon_{1}^{2}-\varepsilon_{0}^{2}=A^{2} \sigma_{z}^{2}\left\{\cos 2 \varphi_{s}\left[\frac{1}{2}+\frac{e^{-2 k^{2}}}{2}-e^{-\frac{k^{2}}{2}}+\frac{k^{2}}{2} e^{-k^{2}}\right]+1-e^{-\frac{k^{2}}{2}}-\frac{k^{2}}{2} e^{-k^{2}}\right\} \text {. }
$$

For a small dilution, the relative emittance change can be expressed as

$$
\frac{\delta \varepsilon}{\varepsilon_{0}} \equiv \frac{\varepsilon_{1}-\varepsilon_{0}}{\varepsilon_{0}} \approx A^{2} \frac{\sigma_{z}^{2}}{2 \varepsilon_{0}^{2}}\left\{\cos 2 \varphi_{s}\left[\frac{1}{2}+\frac{e^{-2 k^{2}}}{2}-e^{-\frac{k^{2}}{2}}+\frac{k^{2}}{2} e^{-k^{2}}\right]+1-e^{-\frac{k^{2}}{2}}-\frac{k^{2}}{2} e^{-k^{2}}\right\}
$$

\section{Case of the small bunch length}

The variable $k$ introduced in Eq. (5) is the rms bunch length expressed in the phase units, $k \equiv$ $\sigma_{\varphi}$. Typically, this value is small, $\sigma_{\varphi} \ll 1$. For this case, Eq. (16) can be expanded to the first nonzero terms:

$$
\frac{\delta \varepsilon}{\varepsilon_{0}} \approx A^{2} \frac{\sigma_{z}^{2}}{2 \varepsilon_{0}^{2}}\left[\cos ^{2} \varphi_{s}\left(\frac{3}{8} \sigma_{\varphi}^{4}+\frac{19}{48} \sigma_{\varphi}{ }^{6}\right)+\frac{\sigma_{\varphi}{ }^{6}}{6}\right]
$$

For a bunching cavity, $\varphi_{s}=-\frac{\pi}{2}$, Eq. (17) can be transformed to show explicitly the initial parameters as follows:

$$
\frac{\delta \varepsilon}{\varepsilon_{0}} \approx \frac{1}{12}\left(\frac{1}{\beta^{2} \gamma^{3}} \frac{U}{M c^{2}} \cdot \frac{\beta c}{2 \pi f_{c} \varepsilon_{0}}\right)^{2} \sigma_{\varphi}^{8}
$$

For convenience, Eq. (18) can be expressed through the RF wavelength $\lambda=\frac{c}{f_{c}}$ and normalized emittance $\varepsilon_{n}=\beta \gamma^{3} \varepsilon_{0}$ :

$$
\frac{\delta \varepsilon}{\varepsilon_{0}} \approx \frac{1}{12}\left(\frac{U}{M c^{2}} \cdot \frac{\lambda}{2 \pi \varepsilon_{n}}\right)^{2} \sigma_{\varphi}^{8}
$$


For an accelerating cavity, $\left|\varphi_{S}\right|-\frac{\pi}{2} \gg \sigma_{\varphi} \sqrt{\frac{2}{9}}$, Eq. (17) is approximated

$$
\frac{\delta \varepsilon}{\varepsilon_{0}} \approx \frac{3}{16}\left(\frac{U \cos \varphi_{s}}{M c^{2}} \cdot \frac{\lambda}{2 \pi \varepsilon_{n}}\right)^{2} \sigma_{\varphi}{ }^{6} .
$$

Note that in Eq. (20), the synchronous phase enters only as the energy gain by the synchronous particle.

\section{Acknowledgement}

This manuscript has been authored by Fermi Research Alliance, LLC under Contract No. DEAC02-07CH11359 with the U.S. Department of Energy, Office of Science, Office of High Energy Physics. 